\title{
Electrophysiological network alterations in adults with copy number variants associated with high neurodevelopmental risk
}

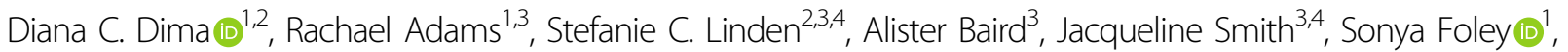 \\ Gavin Perry ${ }^{1}$, Bethany C. Routley ${ }^{1}$, Lorenzo Magazzini ${ }^{1}$, Mark Drakesmith $\mathbb{B}^{1,2}$, Nigel Williams $s^{2,3,4}$, Joanne Doherty ${ }^{1,3,4}$, \\ Marianne B. M. van den Bree ${ }^{2,3,4}$, Michael J. Owen $\mathbb{B}^{2,3,4}$, Jeremy Hall $\mathbb{D}^{2,3,4}$, David E. J. Linden ${ }^{1,2,3,4}$ and Krish D. Singh (1)
}

\begin{abstract}
Rare copy number variants associated with increased risk for neurodevelopmental and psychiatric disorders (referred to as ND-CNVs) are characterized by heterogeneous phenotypes thought to share a considerable degree of overlap. Altered neural integration has often been linked to psychopathology and is a candidate marker for potential convergent mechanisms through which ND-CNVs modify risk; however, the rarity of ND-CNVs means that few studies have assessed their neural correlates. Here, we used magnetoencephalography (MEG) to investigate resting-state oscillatory connectivity in a cohort of 42 adults with ND-CNVs, including deletions or duplications at 22q11.2, 15q11.2, 15q13.3, 16p11.2, 17q12, 1q21.1, 3q29, and 2p16.3, and 42 controls. We observed decreased connectivity between occipital, temporal, and parietal areas in participants with ND-CNVs. This pattern was common across genotypes and not exclusively characteristic of 22q11.2 deletions, which were present in a third of our cohort. Furthermore, a datadriven graph theory framework enabled us to successfully distinguish participants with ND-CNVs from unaffected controls using differences in node centrality and network segregation. Together, our results point to alterations in electrophysiological connectivity as a putative common mechanism through which genetic factors confer increased risk for neurodevelopmental and psychiatric disorders.
\end{abstract}

\section{Introduction}

A number of rare genetic variants occurring through the deletion or duplication of chromosomal segments are associated with significantly increased risk for a range of neurodevelopmental disorders (ND), including schizophrenia, autism spectrum disorder (ASD), and developmental delay ${ }^{1}$. Although the underlying mechanisms remain poorly understood, these copy number variants (referred to hereafter as ND-CNVs) are thought to increase the risk for psychopathology through alterations

\footnotetext{
Correspondence: Diana C. Dima (DimaDC@cardiff.ac.uk)

${ }^{1}$ Cardiff University Brain Research Imaging Centre (CUBRIC), School of Psychology, Cardiff University, Maindy Road, Cardiff CF24 4HQ, UK

${ }^{2}$ Neuroscience and Mental Health Research Institute (NMHRI), Cardiff University, Maindy Road, Cardiff CF24 4HQ, UK

Full list of author information is available at the end of the article
}

in neural structure and function. Thus, neuroimaging studies in participants with ND-CNVs provide a unique opportunity to study intermediate phenotypes of mental disorders.

Recent work suggests that CNV-specific phenotypic outcomes are limited, pointing instead to a large degree of similarity across phenotypes associated with different ND$\mathrm{CNVs}^{2,3}$. Focusing on convergent neural alterations across different genotypes can thus help elucidate the mechanisms linking ND-CNVs at different loci to a shared psychopathology and increase in neurodevelopmental risk.

Failures of functional neural integration have long been considered a hallmark of neurodevelopmental disorders such as schizophrenia ${ }^{4,5}$. In recent years, evidence of disrupted connectivity has also emerged in ASD 
populations $^{6,7}$ and has been shown to transcend diagnostic boundaries ${ }^{8}$. ND-CNVs are thought to increase disorder risk by acting on large-scale neural integration through molecular and cellular mechanisms ${ }^{9}$. Studying functional network alterations in participants with NDCNVs could thus help establish their reliability as biomarkers of neurodevelopmental risk. Synchronous oscillatory activity thought to support communication between brain areas is of particular interest as a potential biomarker of neurodevelopmental risk, and can be measured at rest using electro- and magneto-encephalography (EEG/MEG).

However, the rarity of ND-CNVs means that evidence of their functional connectivity correlates is scarce. Of the genetic imaging studies conducted so far, most have focused on the 22q11.2 deletion syndrome. This deletion is associated with a number of physical phenotype manifestations as well as high risk for psychopathology ${ }^{10-13}$. The presence of a 22q11.2 deletion has been linked to alterations in brain structure and function ${ }^{14-17}$, including disrupted structural connectivity ${ }^{18,19}$. Although fewer studies have investigated functional connectivity, they report similarly disrupted networks using functional $\mathrm{MRI}^{20-22}$ and $\mathrm{EEG}^{23}$.

Despite emerging evidence of white matter alterations associated with other $\mathrm{ND}-\mathrm{CNVs}^{24-26}$, very few studies have investigated their functional correlates. Recent electrophysiological research reported increased betaband activity in participants with 15q11.2-q13.1 duplications $^{27,28}$ and 16p11.2 deletions ${ }^{29}$, as well as delayed evoked responses in the latter ${ }^{24,30}$. Based on current evidence it is difficult to assess the extent of functional connectivity alterations, especially for rarer ND-CNVs.

To address this, we investigated oscillatory connectivity measured with MEG in participants with ND-CNVs at nine different loci. Given the common phenotypic outcomes associated with $\mathrm{ND}-\mathrm{CNVs}^{2}$, this approach can identify convergent endophenotypes of potentially higher clinical relevance. Because a third of our cohort had $22 q 11.2$ deletions, we also investigated alterations in connectivity separately in this subgroup and in the group of participants with other ND-CNVs. This allowed us to assess the specificity of the effects, especially considering previous findings of widespread neural alterations associated with 22q11.2 deletions.

In both subgroups, we found evidence of disrupted alpha and beta-band oscillatory connectivity in posterior brain regions. Furthermore, using graph theory measures of network topology and information transfer, we were able to identify participants with ND-CNVs based on their individual connectivity maps. The two approaches highlighted common patterns of dysconnectivity in participants with ND-CNVs, as well as specific network features that might be linked to CNV pathogenicity.

\section{Materials and methods \\ Participants}

MEG data were acquired in 42 adults with ND-CNVs targeted for their high penetrance for neurodevelopmental disorders ( 22 female; mean age $38.5 \pm 12.5$ years; range 19-76). ND-CNVs at nine different loci were represented in the cohort, with 14 (33\%) participants carrying 22q11.2 deletions (Table 1).

Recruitment was performed through NHS genetics clinics and relevant support groups within the UK. Written consent was obtained in accordance with The Code of Ethics of the World Medical Association (Declaration of Helsinki), and all procedures were approved by the South East Wales Research Ethics Committee. Informed consent was obtained from all subjects.

Table 1 Participant information (ND-CNV status, demographic information, number of psychiatric diagnoses, and IQ).

\begin{tabular}{|c|c|c|c|c|c|c|c|}
\hline CNV and critical region (hg19) & Total $N$ & $N$ female & Age (mean $\pm S D)$ & $N$ diag $($ mean $\pm \mathrm{SD})$ & VIQ (mean \pm SD) & $\mathrm{PIQ}($ mean $\pm \mathrm{SD})$ & FSIQ $($ mean \pm SD) \\
\hline All CNV & 42 & 22 & $38.53 \pm 12.55$ & $2.93 \pm 2.54$ & $88.72 \pm 18.84$ & $96.63 \pm 14.63$ & $91.24 \pm 17.15$ \\
\hline 22q11.2del chr22:19,037,332-21,466,726 & 14 & 9 & $38.97 \pm 16.43$ & $3 \pm 2.07$ & $86.28 \pm 21.23$ & $95.28 \pm 15.6$ & $89.93 \pm 17.88$ \\
\hline 22q11.2dup chr22:19,037,332-21,466,726 & 4 & 13 & $38.32 \pm 10.44$ & $2.89 \pm 2.78$ & $90 \pm 17.77$ & $97.33 \pm 14.36$ & $91.89 \pm 17.08$ \\
\hline 17q12dup chr17:34,815,904-36,217,432 & 2 & & & & & & \\
\hline 16p11.2del chr16:29,650,840-30,200,773 & 1 & & & & & & \\
\hline 16p11.2dup (distal) chr16:28,823,196-29,046,783 & 1 & & & & & & \\
\hline 15q13.1-13.3del (BP4-5) chr15:31,080,645-32,462,776 & 2 & & & & & & \\
\hline 15q13.1-13.3dup (BP4-5) chr15:31,080,645-32,462,776 & 1 & & & & & & \\
\hline 15q11.2del (BP1-2) chr15:22,805,313-23,094,530 & 7 & & & & & & \\
\hline 15q11.2dup(BP1-2) chr15:22,805,313-23,094,530 & 1 & & & & & & \\
\hline 15q11.2q12dup(BP2-3; PWS/AS) chr15:22,805,313-28390339 & 1 & & & & & & \\
\hline 3q29del chr3:195,720,167-197,354,826 & 1 & & & & & & \\
\hline 2p16.3del (NRXN1) chr2:50145643-51259674 & 1 & & & & & & \\
\hline 1q21.1 del chr1:146,527,987-147,394,444 & 5 & & & & & & \\
\hline 1q21.1dup chr1:146,527,987-147,394,444 & 1 & & & & & & \\
\hline Controls & 42 & 22 & $33.35 \pm 9.58$ & - & - & - & - \\
\hline
\end{tabular}

Note that the 22q11.2 deletion group included two atypical deletions and one adjacent deletion. 
All participants with ND-CNVs were assessed using the Psychiatric Assessment Schedule for Adults with Developmental Disability (PAS-ADD; Moss et al. ${ }^{31}$ ). Twentyfour participants were also assessed using the Structured Interview for Prodromal Symptoms (SIPS; Miller et al. ${ }^{32}$ ), and 26 participants using the Structured Clinical Interview for DSM (SCID II; First and Gibbon ${ }^{33}$ ). Diagnoses were assigned by research psychologists and verified by an adult psychiatrist using the Diagnostic and Statistical Manual for Mental Disorders, 4th and 5th Editions. Of the 42 participants, 34 had at least one diagnosis; 22 had anxiety disorders, 15 had mood disorders, and 15 had neurodevelopmental disorders (including six with intellectual disability and six with autism spectrum disorders). Five participants exhibited full or attenuated psychotic symptoms, of whom four met the criteria for a psychotic disorder, with two schizophrenia diagnoses in the 22q11.2 deletion group. There was no significant difference in number of diagnoses between the 22q11.2 deletion group and the other ND-CNV group $(t(33.7)=0.14, P=0.89)$.

IQ tests were administered using the Wechsler Adult Intelligence Scale (WAIS-III). We report verbal IQ (VIQ), performance IQ (PIQ) and full scale IQ scores (FSIQ). There was no significant difference in IQ between the 22q11.2 deletion group and the other ND-CNV group $(t$ $(22.7-25.1)<0.57, P>0.58)$.

In the ND-CNV group, $62 \%$ of participants were taking medication for physical, neurological or mood disorders (e.g., high blood pressure, asthma, pain/migraine, and depression/anxiety), with the most common medications including gabapentin, co-codamol (combination of codeine and paracetamol), and fluoxetine $(N=3)$. Given the high variability of medications, their effects could not be systematically investigated; however, their impact was alleviated in MEG analysis by tests of generalizibility (e.g., resampling).

Controls were selected among resting-state datasets acquired at CUBRIC as part of the "100 Brains"' and "UK MEG Partnership" projects. These cohorts included healthy participants with no history of neurological or neuropsychiatric disorders, and 42 controls were chosen to match the gender and age of the ND-CNV carriers as closely as possible ( 22 female; mean age $33.3 \pm 9.6$ years; range 22-71). These measurements were acquired under protocols approved by the Cardiff University School of Psychology Ethics Committee, and informed consent was obtained from all participants.

Since a third of the ND-CNV cohort consisted of participants with 22q11.2 deletions, we assessed the impact of this subgroup by repeating all analyses on (1) participants with other ND-CNVs (except 22q11.2 deletions) and their matched controls $(N=56)$, and (2) participants with 22q11.2 deletions and their matched controls $(N=28)$.

\section{Genotyping}

Participants with ND-CNVs were genotyped using the Illumina HumanCoreExome whole genome SNP array, which contained an additional 27,000 genetic variants at loci previously linked to neurodevelopmental disorders, including CNVs. The raw intensity data was processed using Illumina Genome Studio software (version 2011.1). PennCNV (version 1.0.3) was used to perform CNV calling in order to confirm the presence of the ND-CNV in each case sample, with each $\mathrm{CNV}$ being required to span a minimum of ten informative SNPs and to be at least $10 \mathrm{~kb}$ in length. $\mathrm{CNV}$ coordinates were specified according to genome version hg19, and the boundaries of each $\mathrm{CNV}$ were confirmed by manually inspecting the $\log \mathrm{R}$ ratio and $\mathrm{B}$ allele frequency plots at each of the genomic regions of interest (Table 1). Genetic information was not available for control participants; given the rarity of these genotypes in the general population, they were assumed to carry no ND-CNVs.

\section{Data collection}

Five-minute resting-state MEG recordings were made using a 275-channel CTF radial gradiometer system (CTF, Vancouver, Canada) at a sampling rate of $1200 \mathrm{~Hz}$. Three of the sensors were turned off due to excessive noise, and 29 reference channels were recorded to improve noise cancellation $^{34}$. During the recordings, participants were seated upright and fixated their eyes on a red fixation point presented centrally on either a CRT monitor or LCD projector. Three electromagnetic coils were placed at fiducial locations (nasion and pre-auricular) for head localization.

To aid in source localization, structural T1-weighted MRI scans were also acquired using a $3 \mathrm{~T}$ General Electric or Siemens MRI scanner with a $1 \mathrm{~mm}$ isotropic FSPGR/ MPRAGE pulse sequence.

\section{Data analysis \\ Pre-processing}

To remove muscle artifacts, a semiautomatic procedure was implemented using the FieldTrip toolbox ${ }^{35}$ and MATLAB R2015a. Sensor time-series were bandpassfiltered between 110 and $140 \mathrm{~Hz}$ and $z$-transformed; segments exceeding a participant-specific $z$-score threshold were removed. Next, eye movement and cardiac artifacts were projected out of the data using independent component analysis (ICA). Noisy channels exhibiting high variance were also removed from the data where necessary. There was no significant difference in recording duration after artifact rejection between the ND-CNV and control groups $(t(81.8)=1.61, P=0.11$, mean duration $255.88 \pm 29.31 \mathrm{~s}$ and $245.33 \pm 30.86 \mathrm{~s}$, respectively).

Head motion was monitored continuously in 18/42 ND$\mathrm{CNV}$ datasets and 40/42 control datasets, and head 
localization was performed at the start and end of the recording in the remaining datasets. There was no significant difference between the ND-CNV and control groups in maximum head coil displacement between the beginning and end of the recording $(t(79.8)=0.85, P=$ 0.39 , mean displacement $2.07 \pm 3.62 \mathrm{~mm}$ and $2.7 \pm$ $3.06 \mathrm{~mm}$, respectively). In datasets with continuous head localization, the maximum distance of the head coils from their average position across the entire recording did not significantly differ between groups $(t(39.4)=1.44, P=$ 0.16 , mean distance $4.74 \pm 3.5 \mathrm{~mm}$ and $3.21 \pm 4.2 \mathrm{~mm}$, respectively).

Prior to source localization, coregistration was performed by manually marking head coil locations on each participant's MRI using FieldTrip. The data were downsampled to $600 \mathrm{~Hz}$ and bandpass-filtered in six different frequency bands: delta $(2-4 \mathrm{~Hz})$, theta $(4-8 \mathrm{~Hz})$, alpha $(8-13 \mathrm{~Hz})$, beta $(13-30 \mathrm{~Hz})$, low gamma $(40-60 \mathrm{~Hz})$, and high gamma $(60-90 \mathrm{~Hz})$.

\section{Estimating functional connectivity}

To assess group differences in resting-state connectivity (Fig. 1), we focused on amplitude-amplitude coupling of source-localized oscillatory signals ${ }^{36}$. Continuous data in each of the six frequency bands were projected into source space using a linearly constrained minimum variance (LCMV) beamformer. Sources were reconstructed on a $6 \mathrm{~mm}$ template grid warped to each participant's MRI, using a multiple local-spheres forward model ${ }^{37}$. To alleviate the depth bias, beamformer weights were normalized by their vector norm ${ }^{38}$.

Next, 90 nodes corresponding to cortical regions of interest (ROI) in the automated anatomical labeling (AAL) atlas ${ }^{39}$ were identified by performing a frequency analysis on all sources within each ROI and selecting the source with the largest temporal standard deviation. Continuous virtual sensor timecourses corresponding to the 90 nodes were then reconstructed and bandpassfiltered into the frequency bands of interest.

To avoid spurious correlations, the node time-series were orthogonalized using a multivariate symmetric orthogonalization approach ${ }^{40}$. A Hilbert transform was used to calculate oscillatory amplitude envelopes, which were then despiked using a median filter, downsampled to $1 \mathrm{~Hz}$, and trimmed to avoid filter and edge effects. To obtain connectivity matrices, pairwise correlations were calculated between the 90 Hilbert envelopes. Next, a Fisher transform was applied to obtain $z$ scores with zero mean and unit variance across connections in each participant's map. This procedure corrected for possible systematic differences across participants, for example due to differences in data quality ${ }^{41}$.

Intracranial volume (ICV), quantified as the number of 1-mm isotropic voxels inside the brain, was smaller in the ND-CNV group than the control group $(t(65.5)=-2.19$,
$P=0.03)$, in line with some previous reports ${ }^{42-44}$. The potential impact of this difference on the MEG results was alleviated through the source localization procedure and the $z$-scoring of the connectivity matrices.

In addition to the six frequency bands listed above, a combined measure of connectivity was obtained by calculating the vector-sum of connectivity matrices across all frequency bands ${ }^{45}$.

\section{Group differences in resting-state connectivity}

To reduce the impact of noise, a conservative ranking procedure ${ }^{45}$ was used to threshold the connectivity maps for the purposes of between-group comparisons. This consisted of calculating the rank of each connection in participantlevel connectivity matrices and averaging the resulting rank map across participants in each group. Only the top 20\% edges in the average rank map were considered "valid" and selected for further analysis. To ensure that large differences in signal across cohorts were not discarded by this procedure, the rank-thresholding procedure was performed separately in each cohort, and connections determined as "valid" in either cohort were included in further analysis.

We report differences between groups using three statistical thresholds. First, Welch's $t$-tests were conducted at each valid edge, and initial patterns were identified using an uncorrected $\alpha=0.05$. Second, correction for multiple comparisons was applied using a randomization procedure with 10,000 sign-shuffling iterations and maximal statistic thresholding (omnibus $\alpha=0.05^{46}$ ).

Third, the robustness of cohort differences was evaluated through a resampling procedure. Increases and decreases in connectivity between groups were tabulated using random samples of half of each group. This was repeated across 10,000 iterations, and edges showing a consistent effect direction across at least $95 \%$ of iterations were considered robust.

Although few edges survived the conservative omnibus correction across individual connections, we based our conclusions on the resampling analysis, which converged with the uncorrected $t$-test results and pointed to robust patterns of differences in connectivity. Furthermore, we performed a decoding analysis using graph theory metrics to assess the discriminating information present at the network level (see below).

To control for potential confounds (for example, resulting from imperfect age matching between the ND$\mathrm{CNV}$ and control groups), an additional multiple regression analysis was performed. Combined-frequency connectivity matrices were entered as response variables with a categorical predictor (ND-CNV presence) and three covariates (age, gender, and ICV). A resampling procedure as described above was performed to assess the robustness of between-group differences. The sign of the regression slope associated with the main predictor was 


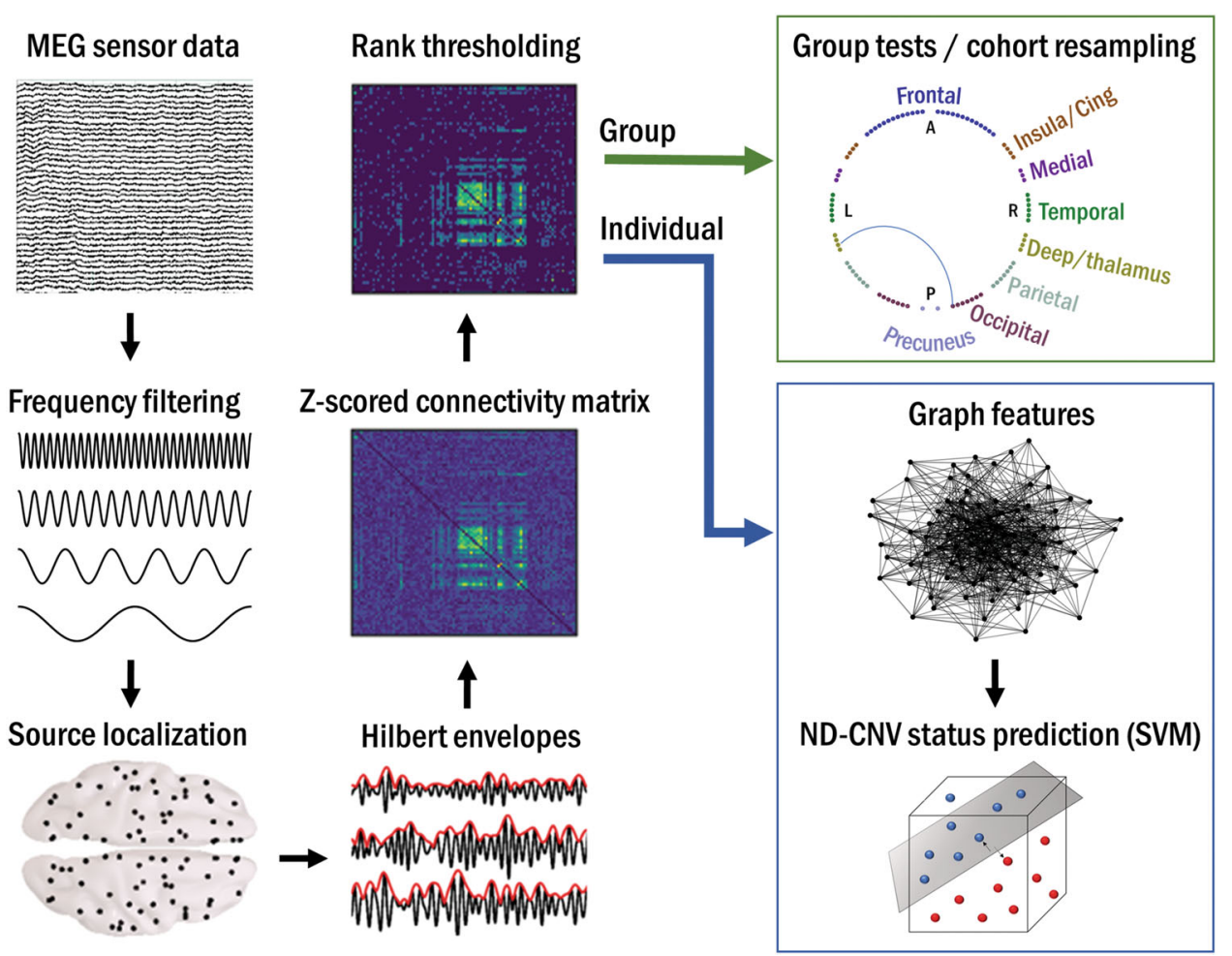

Fig. 1 Overview of the analysis pipeline. Resting-state MEG data were preprocessed, filtered into six frequency bands, and projected into source space. Hilbert envelopes were calculated at 90 AAL-atlas-based virtual sensor locations, and correlated to obtain functional connectivity matrices. These were $z$-scored and rank-thresholded at the group level for between-group analyses, and at the subject level for data-driven prediction of NDCNV status using graph theory.

tabulated across 1000 split-half cohort randomizations. Edges showing consistent effects across $95 \%$ of iterations were considered robust.

This is an exploratory study with a limited sample size due to the rarity of ND-CNVs. Although we are not aware of similar MEG investigations, an EEG study in participants with 22q11.2 $\mathrm{DS}^{23}$ found resting-state connectivity effect sizes ranging from moderate to large. Our sample size can detect a large effect (Cohen's $d=0.8$ ) with $95 \%$ power and a moderate effect $(d=0.5)$ with $62 \%$ power according to a post-hoc power analysis.

\section{Individual networks: identifying participants with ND-CNVs using graph theory}

Next, a data-driven graph theory approach was used to assess whether participants with ND-CNVs could be distinguished from unaffected controls using functional connectivity features. To this aim, the cohort was divided into training and test sets using an iterated crossvalidation procedure.

This analysis focused on individual networks by selecting the top $20 \%$ of connections in each participant's normalized connectivity map as the basis for undirected graphs. This approach avoided bias by ensuring the independence of training and test sets, while at the same time allowing us to detect any informative differences in connection strength. Networks were then characterized using six nodal graph theory metrics. These included three measures of node connectedness: degree (the number of connections linking each node to other nodes); betweenness centrality (the fraction of shortest paths between nodes containing a given node); and eccentricity (the maximal shortest path from a node to any other node). Global efficiency (the average inverse shortest path between a node and all others) was evaluated as a measure of network integration. Finally, two metrics captured network modularity: local efficiency (the average inverse shortest path between a node and its neighbors) and clustering coefficient (the fraction of connected node triplets around a node). All metrics with the exception of node degrees were weighted by the inverse of the normalized connectivity matrices; in other words, stronger amplitude-amplitude coupling was taken to reflect shorter paths between nodes. Graph theory analyses were performed using the graph and network algorithms in MATLAB R2019a and the Brain Connectivity Toolbox ${ }^{47}$.

To discriminate between groups, a linear support vector machine (SVM) classifier ${ }^{48}$ was trained on each of the 
node metrics. Additionally, a pooled feature vector was created by combining the six metrics to maximize the amount of complementary information input to the classifier. This approach makes use of information across all nodes, while avoiding the need for multiple testing.

Classification was performed between the ND-CNV and control groups, as well as between the two ND-CNV subgroups (22q11.2 deletions and other ND-CNVs) and their matched controls. To avoid overfitting, model performance was evaluated using 100 iterations of stratified five-fold cross-validation. This entailed iteratively leaving out a fifth of the data for testing and training the model on the remaining data, whilst ensuring balanced group representation in each fold. Performance was quantified using accuracy (proportion correctly classified observations), sensitivity (true positive rate) and specificity (true negative rate) in order to highlight any asymmetries in $\mathrm{ND}-\mathrm{CNV}$ and control identification. Furthermore, significance was assessed by shuffling the true labels 5000 times and recomputing classifier accuracy to estimate the empirical chance level and calculate a one-tailed $p$-value ${ }^{46}$.

\section{Results}

\section{Connectivity alterations associated with ND-CNVs}

The analysis of group differences in oscillatory connectivity revealed the largest number of valid connections (exceeding a rank of 0.8 in group average maps) in the alpha and beta bands (Fig. 2). The uncorrected $t$-test showed a pattern of decreases in oscillatory connectivity between posterior, parietal and temporal nodes in the ND-CNV group, with the exception of a few righthemisphere edges. More extensive cohort effects were detected using the combined frequency maps (61 edges exceeded the uncorrected threshold, compared to 1, 28, and 42 in the theta, alpha, and beta bands). These patterns were robust to random sub-sampling of the cohort, suggesting that they were not driven by individual subjects. Most connections did not survive omnibus correction for multiple comparisons at the individual edge level, with the exception of a small number of left-hemisphere connections, including the precuneus, early visual cortex, and parietal regions.

Importantly, a similar pattern of hypoconnectivity was observed even after excluding participants with 22q11.2 deletions and their matched controls (Fig. 2b, c). Both ND-CNV subgroups showed decreased posterior connectivity (Fig. 3; 18 connections decreased in both groups), indicating that the overall pattern was not driven by the 22q11.2 deletion group.

This pattern occurred despite higher heterogeneity in the mixed ND-CNV group (Supplementary Fig. 1) and might thus reflect convergent alterations across genotypes. On the other hand, participants with 22q11.2 deletions exhibited more right-hemisphere hyperconnectivity compared to controls. These effects spanned the precuneus and parietal cortex, as well as frontal regions, suggesting some overlap with the default mode network (DMN).

To ensure that these differences were not affected by potential confounds, the cohort resampling tests on combined frequency maps were repeated as a multiple linear regression with age, gender, and ICV as covariates. This analysis revealed fewer connections (65 compared to the original 92 in the whole cohort analysis), but largely similar patterns of dysconnectivity (Fig. 3b).

Furthermore, although IQ scores could not be included in this analysis because they were not available for the control group, IQ scores in the ND-CNV group significantly correlated with connectivity strength at only four edges (Supplementary Fig. 2).

\section{Network features as predictors of ND-CNV status}

A graph theory framework was employed to identify participants with ND-CNVs from their functional networks based on combined frequency maps. This approach has the advantage of reducing dimensionality and complements the edge-focused group testing approach described above. Despite methodological differences between the two analyses, a visualization of the nodal graph theory features shows overlap with the nodes highlighted in the group analysis (Supplementary Figs. 3-6).

Graph theory metrics were successful at identifying ND$\mathrm{CNV}$ participants relative to unaffected controls. The best prediction accuracy was achieved by combining the six node features (Supplementary Table 1 and Fig. 4a; maximum accuracy $71.4 \% \pm 2.98, p=0.0002$ ). However, participants with 22q11.2 deletions were more consistently correctly classified than those with other ND-CNVs (Fig. 4b).

This was confirmed by subgroup classification analyses, which also pointed to subgroup differences. When excluding participants with 22q11.2 deletions, the best decoding accuracies were achieved using node eccentricities $(65.02 \% \pm 4.28, p=0.0022)$, node degrees, and a joint feature model. On the other hand, all node features were successful in discriminating participants with 22q11.2 deletions from their matched controls, with the best performance obtained using the clustering coefficient $(89.02 \% \pm 4, p=0.0002)$. Furthermore, removing the connectivity map thresholding step from this analysis did not significantly affect the results. This suggests that successful classification is not exclusively driven by differences in edge selection (Supplementary Fig. 7).

These results point to commonalities in network features (such as decreased centrality) that allow for the successful classification of participants with ND-CNVs across distinct genotypes. On the other hand, the features are specific enough to allow successful discrimination between participants with 22q11.2 deletions and other ND-CNVs (Supplementary Table 1). 


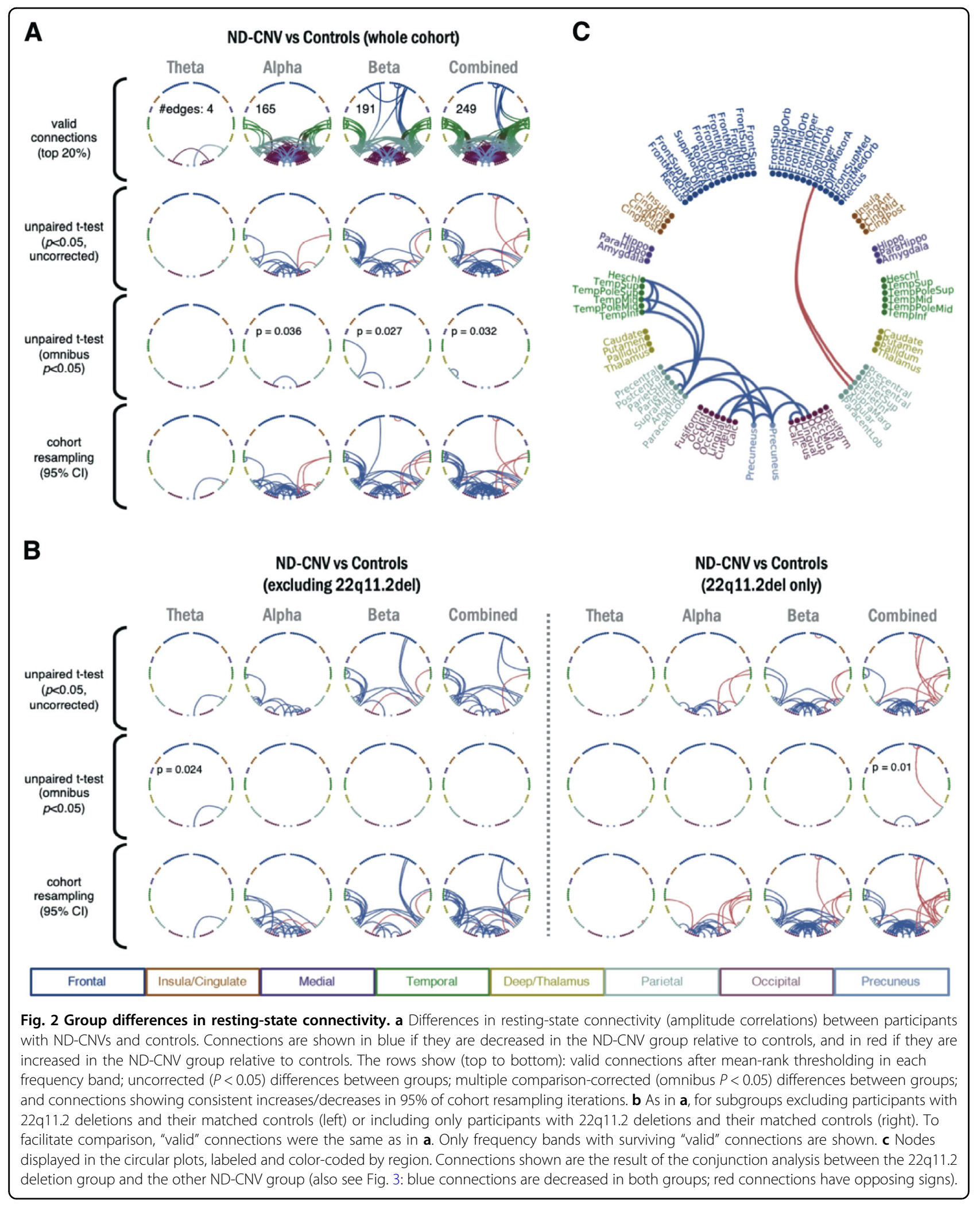




ND-CNV vs Controls
(Whole cohort)
(excluding 22q11.2del)
Fig. 3 Differences in connectivity are not driven by age, gender, and intracranial volume. Connections meeting the 95\% confidence criterion
in the cohort resampling test are displayed for all group tests (first three columns). Connections are shown in blue if they are decreased in the ND-
CNV group relative to controls, and in red if they are increased in the ND-CNV group relative to controls. The last column shows supra-threshold
connections in both the 22q11.2 deletion group and the other ND-CNV group; here, connections are shown in blue if they are decreased in both
groups, and in red if they have opposite signs. Line width increases with effect robustness. a Connections exhibiting robust differences based on the
cohort resampling test of combined frequency matrices, plotted on the template brain. b As in a, after including age, gender, and intracranial volume
as covariates in a multiple linear regression with "ND-CNV presence" as a main categorical predictor.

Given the higher overall burden of 22q11.2 deletions in neurodevelopmental disorders ${ }^{1}$, this suggests that increased neurodevelopmental risk may be associated with more salient alterations in network function and may underpin specific genotype effects.

\section{Discussion}

To our knowledge, the present study provides the first insight into oscillatory connectivity alterations in people with rare ND-CNVs. Using both an established group analysis pipeline and a data-driven graph theory framework, we uncovered a pattern of functional dysconnectivity affecting posterior regions in participants with NDCNVs. Although individual connection effect sizes were generally low, these patterns were robust to effects of age, gender, and intracranial volume, and emerged despite a conservative thresholding approach restricted to the most reproducible connections. These patterns also allowed for the successful classification of participants with NDCNVs using graph theory metrics.

Effects originated in the alpha and beta frequency bands, which are thought to underpin long-range communication between brain areas ${ }^{49}$. Connections linking parietal, temporal, and occipital areas were most consistently affected in both the 22q11.2 deletion group and the other ND-CNV group. Similar patterns have been previously reported in schizophrenia patients $^{50}$, including alpha-band parietal hypoconnectivity in first-episode schizophrenia ${ }^{51}$. Furthermore, posterior structural network alterations have been identified as an early marker of $\mathrm{ASD}^{52}$, suggesting a link between such alterations and increased neurodevelopmental risk.

Similar connectivity changes in the visual processing system and the default mode network have been shown in people with 22q11.2 deletions using structural and functional MRI ${ }^{53-55}$. Here, we found that these effects are not restricted to the 22q11.2 deletion group, suggesting that long-range connectivity could act as a common marker across genetic variants. Although noninvasive measurements cannot provide direct mechanistic insight, this is consistent with potential alterations in excitatory-inhibitory balance ${ }^{56,57}$ as a mechanism for pleiotropic genetic effects underlying neurodevelopmental disorders ${ }^{58-60}$. This is thought to occur through increased excitation or disinhibition caused by gene haploinsufficiency and mediated by impaired GABA and NMDA receptor function ${ }^{61-63}$.

Despite sample size limitations, differences between the two subgroups also point to effects specific to the highly penetrant 22q11.2 deletions. Hypoconnectivity was more extensive in people with other ND-CNVs, while the 22q11.2 deletion group exhibited more focused patterns; these were robust to cohort resampling, suggesting that they are unlikely to be driven by individual cases. These differences were reflected in the graph theory analysis. Although all network features were altered in the 22q11.2 deletion group, their increased modularity was particularly discriminative, in line with previous reports of increased structural network segregation in people with $22 q 11.2$ deletions ${ }^{18,22,64}$. For other ND-CNVs, the only predictive features were centrality measures (specifically the node eccentricity and degree), reflecting hypoconnectivity in the ND-CNV cohort. Between and withingroup classification results (Supplementary Table 1) highlight the ability of graph theory metrics to capture both convergent and specific network alterations, which could help elucidate the link between $\mathrm{CNV}$ pathogenicity and neural system function. 

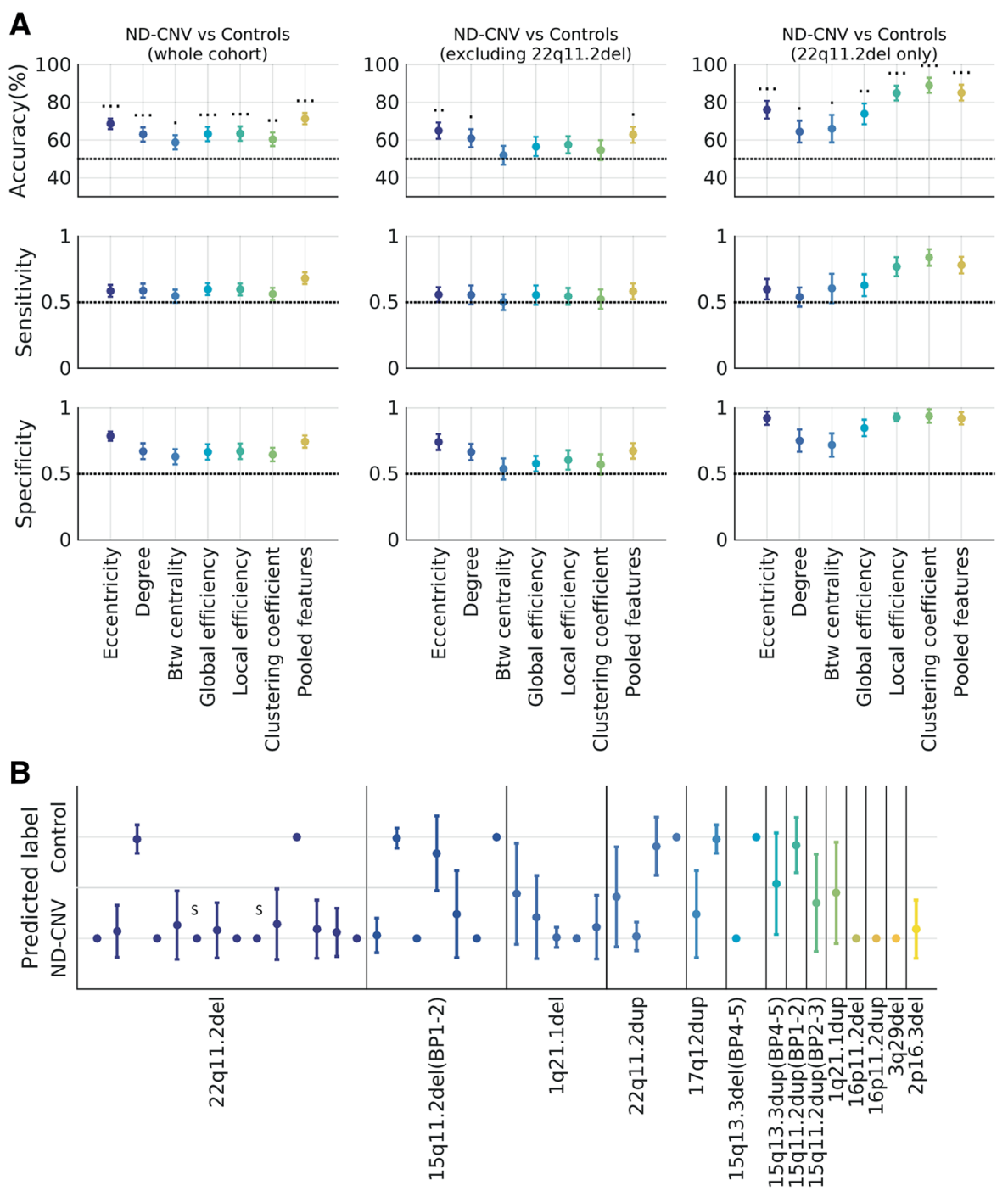

Fig. 4 Classifying participants with ND-CNVs and unaffected controls from individual MEG functional networks using graph theory. a Classification performance for the three groups, using different metrics to characterize the networks (eccentricity, degree, betweenness centrality, global and local efficiency, clustering coefficient, and a pooled model combining all features). Above-chance accuracies (permutation testing) are marked with 1, 2, and 3 dots respectively for $p<0.05, p<0.01$, and $p<0.001$. $\mathbf{b}$ How well are different ND-CNVs classified? The plot shows the mean predicted label for each of the 42 participants with ND-CNVs across 100 cross-validation iterations using pooled node features. Participants with 22q11.2 deletions are most consistently correctly identified. Two participants with schizophrenia diagnoses are marked with "S". All error bars are \pm SD.

We alleviated concerns of potential systematic betweengroup differences unrelated to genotype by using conservative approaches (e.g., rejecting weaker connections that may introduce noise) and resampling procedures. Despite generally low effect sizes which did not survive an omnibus threshold across individual connections, we base our conclusions on the robustness of the connectivity patterns and their convergence across analyses. Although head motion did not appear to differ between groups, this was not continuously measured in all participants and so we could only partially rule out its effects.
Although the present study was able to evaluate ND$\mathrm{CNV}$ effects independently of the contribution of highly penetrant 22q11.2 deletions, the limited sample size remains a concern common in $\mathrm{CNV}$ imaging research. The high genotype variability within the cohort makes the specificity of these effects difficult to assess, particularly with regard to differences between the 22q11.2 deletion group and other ND-CNVs. Variability within the heterogeneous ND-CNV group was higher than within the 22q11.2 deletion group (Supplementary Fig. 1), suggesting that our focus on convergent alterations may obscure 
specific effects. However, the fact that we see differences robust to resampling in this group, despite its heterogeneity, points to common connectivity alterations across distinct genotypes. Studies recruiting larger samples of participants with ND-CNVs, for example through large multisite collaborations, are necessary to evaluate the generalizability of connectivity patterns and graph theory metrics as "fingerprints" associated with ND-CNV status.

In summary, the present study assessed oscillatory longrange connectivity as a potential marker of pathogenic genetic effects across a range of rare ND-CNVs. Occipital, parietal, and temporal brain areas were characterized by consistent hypoconnectivity across genotypes, which was not exclusively driven by the presence of a large number of participants with highly-penetrant 22q11.2 deletions. Functional networks in the ND-CNV group exhibited decreased node centrality and alterations in network efficiency and structure. Furthermore, features specific to highly penetrant variants were present alongside convergent network alterations and enabled successful NDCNV classification. These results are consistent with a common mechanism for genetic risk, based on an altered balance between excitatory and inhibitory synaptic processes and leading to network dysfunction. We propose that these functional connectivity alterations are an intermediate phenotype on the pathway from synaptic molecular changes to disruption of cognitive function and psychotic illness.

\section{Acknowledgements}

The authors wish to thank: Ffion Evans and Kali Barawi for assistance with psychometric and clinical data collection; Eirini Messaritaki for helpful comments on the graph theory analysis; George Kirov for help with genetic data; and Alexander Shaw for the SourceMesh visualization toolbox. This work was supported by a Wellcome Trust Strategic Award (100202/Z/12/Z), the MRC UK MEG Partnership Grant (MR/K005464/1), and the MRC Doctoral Training Grant (MR/K501086/1)

\section{Author details}

${ }^{1}$ Cardiff University Brain Research Imaging Centre (CUBRIC), School of Psychology, Cardiff University, Maindy Road, Cardiff CF24 4HQ, UK. ${ }^{2}$ Neuroscience and Mental Health Research Institute (NMHRI), Cardiff University, Maindy Road, Cardiff CF24 4HQ, UK. ${ }^{3}$ Division of Psychological Medicine and Clinical Neurosciences, School of Medicine, Cardiff University, Cardiff CF24 4HQ, UK. ${ }^{4}$ MRC Centre for Neuropsychiatric Genetics and Genomics, School of Medicine, Cardiff University, Maindy Road, Cardiff CF24 $4 \mathrm{HQ}$, UK. ${ }^{5} \mathrm{School}$ of Mental Health and Neuroscience, Faculty of Health, Medicine and Life Sciences, Maastricht University, Maastricht, The Netherlands

\section{Code availability}

The resting-state connectivity analysis was performed using Matlab in-house scripts and results were plotted using the SourceMesh toolbox (https://github. com/alexandershaw4/SourceMesh). The graph theory analysis was performed using using the graph and network algorithms in MATLAB R2019a and the Brain Connectivity Toolbox ${ }^{47}$. Decoding analyses were performed using inhouse Matlab scripts (https://github.com/dianadima/mvpa-for-meg) and the LibLinear library ${ }^{65}$. All custom code is available upon request.

\section{Conflict of interest}

The authors declare that they have no conflict of interest.

\section{Publisher's note}

Springer Nature remains neutral with regard to jurisdictional claims in published maps and institutional affiliations.

Supplementary Information accompanies this paper at (https://doi.org/ 10.1038/s41398-020-00998-w).

Received: 3 March 2020 Accepted: 4 September 2020

Published online: 21 September 2020

\section{References}

1. Kirov, G. et al. The penetrance of copy number variations for schizophrenia and developmental delay. Biol. Psychiatry 75, 378-385 (2014).

2. Chawner, S. J. R. A. et al. Genotype-phenotype associations in children with copy number variants associated with high neuropsychiatric risk in the UK (IMAGINE-ID): a case-control cohort study. Lancet Psychiatry 6, 493-505 (2019).

3. Niarchou, M. et al. Psychiatric disorders in children with $16 \mathrm{p} 11.2$ deletion and duplication. Transl. Psychiatry 9, 8 (2019).

4. Friston, K., Brown, H. R., Siemerkus, J. \& Stephan, K. E. The dysconnection hypothesis. Schizophr. Res. 176, 83-94 (2016).

5. Dong, D., Wang, Y., Chang, X., Luo, C. \& Yao, D. Dysfunction of large-scale brain networks in schizophrenia: a meta-analysis of resting-state functional connectivity. Schizophr. Bull. 44, 168-181 (2018).

6. Hull, J. V. et al. Resting-state functional connectivity in autism spectrum disorders: a review. Front. Psychiatry 7, 205 (2017).

7. O'Reilly, C., Lewis, J. D. \& Elsabbagh, M. Is functional brain connectivity atypical in autism? A systematic review of EEG and MEG studies. PLOS ONE 12, e0175870 (2017).

8. Sha, Z., Wager, T. D., Mechelli, A. \& He, Y. Common dysfunction of large-scale neurocognitive networks across psychiatric disorders. Biol. Psychiatry $\mathbf{8 5}$, 379-388 (2019).

9. Karayiorgou, M., Simon, T. J. \& Gogos, J. A. 22q11.2 microdeletions: linking DNA structural variation to brain dysfunction and schizophrenia. Nat. Rev. Neurosci. 11, 402-416 (2010).

10. Jonas, R. K. Montojo, C. A. \& Bearden, C. E. The 22q11.2 deletion syndrome as a window into complex neuropsychiatric disorders over the lifespan. Biol. Psychiatry 75, 351-360 (2014).

11. Schneider, M. et al. Psychiatric disorders from childhood to adulthood in 22q11.2 deletion syndrome: results from the International Consortium on brain and behavior in 22q11.2 deletion syndrome. Am. J. Psychiatry 171, 627-639 (2014).

12. Niarchou, M. et al. Psychopathology and cognition in children with $22 \mathrm{q} 11.2$ deletion syndrome. Br. J. Psychiatry 204, 46-54 (2014).

13. Owen, M. J. \& Doherty, J. L. What can we learn from the high rates of schizophrenia in people with 22q11.2 deletion syndrome? World Psychiatry 15, 23-25 (2016).

14. Zinkstok, J. \& van Amelsvoort, T. A. M. J. Neuropsychological profile and neuroimaging in patients with 22Q11.2 deletion syndrome: a review. Child Neuropsychol. 11, 21-37 (2005).

15. Boot, E. \& van Amelsvoort, T. A. M. J. Neuroimaging correlates of 22 q11.2 deletion syndrome: implications for schizophrenia research. Curr. Top. Med. Chem. 12, 2303-2313 (2013).

16. Reddaway, J. T. et al. Genomic and imaging biomarkers in schizophrenia. Curr. Top. Behav. Neurosci. 40, 325-352 (2018).

17. Sun, D. et al. Large-scale mapping of cortical alterations in 22q11.2 deletion syndrome: convergence with idiopathic psychosis and effects of deletion size. Mol. Psychiatry 25, 1822-1834 (2020).

18. Ottet, M.C. et al. Graph theory reveals dysconnected hubs in 22q11DS and altered nodal efficiency in patients with hallucinations. Front. Hum. Neurosci. 7, 402 (2013).

19. Villalón-Reina, J. E. et al. Altered white matter microstructure in 22q11. 2 deletion syndrome: a multisite diffusion tensor imaging study. Mol. Psychiatry 1-14 (2019)

20. Debbané, M. et al. Resting-state networks in adolescents with 22q11.2 deletion syndrome: associations with prodromal symptoms and executive functions. Schizophr. Res. 139, 33-39 (2012).

21. Padula, M. C. et al. Structural and functional connectivity in the default mode network in 22q11.2 deletion syndrome. J. Neurodev. Disord. 7, 23 (2015). 
22. Scariati, E. et al. Large-scale functional network reorganization in 22q11.2 deletion syndrome revealed by modularity analysis. Cortex 82, 86-99 (2016).

23. Tomescu, M. I. et al. Deviant dynamics of EEG resting state pattern in 22 q11.2 deletion syndrome adolescents: a vulnerability marker of schizophrenia? Schizophr. Res. 157, 175-181 (2014).

24. Berman, J. I. et al. Relationship between M100 auditory evoked response and auditory radiation microstructure in 16p11.2 deletion and duplication carriers. Am. J. Neuroradiol. 37, 1178-1184 (2016).

25. Drakesmith, M. et al. Genetic risk for schizophrenia and developmental delay is associated with shape and microstructure of midline white-matter structures. Transl. Psychiatry 9, 102 (2019).

26. Silva, A. I. et al. Reciprocal white matter changes associated with copy number variation at 15q11.2 BP1-BP2: a diffusion tensor imaging study. Biol. Psychiatry 85, 563-572 (2019)

27. Frohlich, J. et al. A quantitative electrophysiological biomarker of duplication 15q11.2-q13.1 syndrome. PLOS ONE 11, e0167179 (2016).

28. Frohlich, J. et al. Mechanisms underlying the EEG biomarker in Dup15q syndrome. Mol. Autism 10, 29 (2019).

29. Hinkley, L. B. N. et al. Sensorimotor cortical oscillations during movement preparation in $16 \mathrm{p} 11.2$ deletion carriers. J. Neurosci. 39 $7321-7331$ (2019)

30. Jenkins, J. et al. Auditory evoked M100 response latency is delayed in children with 16p11.2 deletion but not 16p11.2 duplication. Cereb. Cortex 26 1957-1964 (2016).

31. Moss, S. et al. Validity of the PAS-ADD for detecting psychiatric symptoms in adults with learning disability (mental retardation). Soc. Psychiatry Psychiatr. Epidemiol. 32, 344-354 (1997).

32. Miller, T. J. et al. Prodromal assessment with the structured interview for prodromal syndromes and the scale of prodromal symptoms: predictive validity, interrater reliability, and training to reliability. Schizophr. Bull. 29, 703-715 (2003).

33. First, M. B. \& Gibbon, M. In Comprehensive Handbook of Psychological Assessment, Vol. 2. Personality Assessment (eds. Hilsenroth, M. J. \& Segal, D. L.). 134-143 (Wiley, Hoboken, NJ, 2004).

34. Vrba, J. Magnetoencephalography: the art of finding a needle in a haystack. Phys. C 368, 1-9 (2002).

35. Oostenveld, R., Fries, P., Maris, E. \& Schoffelen, J. M. FieldTrip: Open source software for advanced analysis of MEG, EEG, and invasive electrophysiological data. Comput. Intell. Neurosci. 2011, 156869 (2011).

36. Colclough, G. L. et al. How reliable are MEG resting-state connectivity metrics? Neuroimage 138, 284-293 (2016).

37. Huang, M. X., Mosher, J. C. \& Leahy, R. M. A sensor-weighted overlappingsphere head model and exhaustive head model comparison for MEG. Phys. Med. Biol. 44, 423-440 (1999).

38. Hillebrand, A., Barnes, G. R., Bosboom, J. L., Berendse, H. W. \& Stam, C. J. Frequency-dependent functional connectivity within resting-state networks: an atlas-based MEG beamformer solution. Neuroimage 59, 3909-3921 (2012).

39. Tzourio-Mazoyer, $\mathrm{N}$. et al. Automated anatomical labeling of activations in SPM using a macroscopic anatomical parcellation of the MNI MRI singlesubject brain. Neuroimage 15, 273-289 (2002).

40. Colclough, G. L., Brookes, M. J., Smith, S. M. \& Woolrich, M. W. A symmetric multivariate leakage correction for MEG connectomes. Neuroimage 117, 439-448 (2015).

41. Siems, M., Pape, A.-A., Hipp, J. F. \& Siegel, M. Measuring the cortical correlation structure of spontaneous oscillatory activity with EEG and MEG. Neuroimage 129, 345-355 (2016)

42. Hu, M.-L. et al. A review of the functional and anatomical default mode network in schizophrenia. Neurosci. Bull. 33, 73-84 (2017).
43. Sønderby, I. E. et al. Dose response of the 16p11.2 distal copy number variant on intracranial volume and basal ganglia. Mol Psychiatry. https://doi.org/ 10.1038/s41380-018-0118-1 (2018).

44. Warland, A., Kendall, K. M., Rees, E., Kirov, G. \& Caseras, X. Schizophreniaassociated genomic copy number variants and subcortical brain volumes in the UK Biobank. Mol. Psychiatry 25, 854-862 (2019).

45. Koelewijn, L. et al. Oscillatory hyperactivity and hyperconnectivity in young APOE- $\varepsilon 4$ carriers and hypoconnectivity in Alzheimer's disease. Elife 8, 1-25 (2019).

46. Nichols, T. E. \& Holmes, A. P. Nonparametric permutation tests for functional neuroimaging: a primer with examples. Hum. Brain Mapp. 25, 1-25 (2001).

47. Rubinov, M. \& Sporns, O. Complex network measures of brain connectivity: uses and interpretations. Neuroimage 52, 1059-1069 (2010).

48. Boser, B. E., Guyon, I. M. \& Vapnik, V. N. A training algorithm for optimal margin classifiers. In: Proceedings of the Fifth Annual Workshop on Computational Learning Theory-COLT'92 (ACM Press, New York, 1992). pp. 144-152.

49. Schnitzler, A. \& Gross, J. Normal and pathological oscillatory communication in the brain. Nat. Rev. Neurosci. 6, 285-296 (2005).

50. Brookes, M. J. et al. A multi-layer network approach to MEG connectivity analysis. Neuroimage 132, 425-438 (2016).

51. Phalen, H., Coffman, B. A., Ghuman, A., Sejdić, E. \& Salisbury, D. F. Non-negative matrix factorization reveals resting-state cortical alpha network abnormalities in the first episode schizophrenia-spectrum. Biol. Psychiatry Cogn. Neurosci. Neuroimaging (2019).

52. Lewis, J. D. et al. Network inefficiencies in autism spectrum disorder at 24 months. Transl. Psychiatry 4, e388-e388 (2014).

53. Scariati, E., Padula, M. C., Schaer, M. \& Eliez, S. Long-range dysconnectivity in frontal and midline structures is associated to psychosis in 22 q11.2 deletion syndrome. J. Neural Transm. 123, 823-839 (2016).

54. Schreiner, M. J. et al. Default mode network connectivity and reciprocal social behavior in 22q11.2 deletion syndrome. Soc. Cogn. Affect. Neurosci. 9, 1261-1267 (2014)

55. Larsen, K. M., Dzafic, I., Siebner, H. R. \& Garrido, M. I. Alteration of functional brain architecture in 22q11.2 deletion syndrome-insights into susceptibility for psychosis. Neuroimage 190, 154-171 (2019).

56. Deco, G. et al. How local excitation-inhibition ratio impacts the whole brain dynamics. J. Neurosci. 34, 7886-7898 (2014).

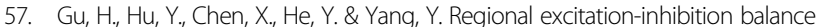
predicts default-mode network deactivation via functional connectivity. Neuroimage 185, 388-397 (2019).

58. Gao, R. \& Penzes, P. Common mechanisms of excitatory and inhibitory imbalance in schizophrenia and autism spectrum disorders. Curr. Mol. Med. 15 146-167 (2015)

59. Lee, E., Lee, J. \& Kim, E. Excitation/inhibition imbalance in animal models of autism spectrum disorders. Biol. Psychiatry 81, 838-847 (2017).

60. Foss-Feig, J. H. et al. Searching for cross-diagnostic convergence: neural mechanisms governing excitation and inhibition balance in schizophrenia and autism spectrum disorders. Biol. Psychiatry 81, 848-861 (2017).

61. Kehrer, C., Maziashvili, N., Dugladze, T. \& Gloveli, T. Altered excitatory-inhibitory balance in the NMDA-hypofunction model of schizophrenia. Front. Mol. Neurosci. 1, 6 (2008)

62. Ramamoorthi, K. \& Lin, Y. The contribution of GABAergic dysfunction to neurodevelopmental disorders. Trends Mol. Med. 17, 452-462 (2011).

63. Pocklington, A. J. et al. Novel findings from CNVs implicate inhibitory and excitatory signaling complexes in schizophrenia. Neuron 86, 1203-1214 (2015).

64. Fan, R.-E., Chang, K.-W., Hsieh, C.-J., Wang, X.-R. \& Lin, C.-J. LIBLINEAR: a library for large linear classification. J. Mach. Learn. Res. 9, 1871-1874 (2008).

65. Sandini, $\mathrm{C}$. et al. Cortical dysconnectivity measured by structural covariance is associated with the presence of psychotic symptoms in 22 q11.2 deletion syndrome. Biol. Psychiatry Cogn. Neurosci. Neuroimaging 3, 433-442 (2018). 